\title{
Denoising of ECG Signals using the Framelet Transform
}

\author{
Marykutty Cyriac \\ Professor, Dept of ECE \\ Jerusalem College of Engineering \\ Chennai, India
}

\author{
Sankar. $P$. \\ Professor, Dept of ECE \\ Jerusalem College of Engineering \\ Chennai, India
}

\begin{abstract}
Denoising of the ECG signals is required, as they are prone to noises during their acquisition. Currently, denoising techniques for ECG signals are mostly available in the wavelet transform domain. In this paper, an approach for denoising the ECG signals in the Framelet domain is proposed. Initially, signals are decomposed using the Framelet transform. After decomposition, they are denoised using a median based thresholding method. The performance evaluation is carried out by comparing the results with that of the wavelet transform.
\end{abstract}

\section{General Terms}

Transforms, Biomedical Application, Signal Processing.

\section{Keywords}

ECG Signal Denoising, Framelet Transform, Thresholding, Wavelet Transform

\section{INTRODUCTION}

The Electrocardiogram (ECG) plays an important role in the process of monitoring and diagnosing cardiac activity or irregularities in the heart rate. Any change in the ECG characteristics and its interval reflects an abnormality in the cardiac condition of a patient. In general, ECG signals are affected by low-frequency and high-frequency interferences. The most common sources of disturbance are baseline drift, power fluctuations, muscular noise and electrode motion artifacts. Power line interferences contain $60 \mathrm{~Hz}$ pickup or 50 $\mathrm{Hz}$ pickup because of improper grounding. Base-line drift is caused in chest-lead ECG signals due to coughing or breathing with large movement of the chest, or when an arm or leg is moved in the case of limb-lead ECG acquisition. Base-line drift can also be caused by the variations in temperature and bias in the instrumentation amplifiers. Motion artifacts are transient baseline changes due to skin impedance with electrode motion. It can generate larger amplitude noisy signals in the ECG waveform. All these undesirable disturbances make the interpretation of the ECG signal difficult and sometimes even impossible.

During recent years, the Discrete Wavelet Transform (DWT) has been proved to be a valuable tool for analysing the data from various domains such as mathematics, science, engineering, economics and also medicine and biology. The wavelet transform has the features that characterize the behavior of ECG signals. Nagendra et al. used the wavelet transform as a tool for processing the non-stationary signals like ECG [1]. The different thresholding methods namely universal, minimax and heuristic methods are used for denoising the ECG signal. Poornachandra and Kumaravel described a sub band adaptive shrinkage method that generalizes hard and soft shrinkages. Wavelet technique was used for decomposing the ECG signal. The proposed sub band adaptive shrinkage is a nonlinear model that works on hyperbolic function, which will outperform the soft shrinkage. This method was then compared with hyper shrinkage, an optimized thresholding scheme based on universal threshold. [2]. David Donoho proposed an approach for the denoising of signals using the soft thresholding function. This method provided better visual quality than procedures based on the mean squared error, termed as the Visushrink [3]. Mikhled Alfaouri and Khaled Daqrouq proposed a method where the signal is decomposed into five levels of the wavelet transform by using Daubechies wavelet and determined a threshold point where minimum error is achieved between the detailed coefficients of the noisy and the original signal. This method was compared with Donoho's method of denoising [4]. The ECG signal can be used for the assessment of mental stress. A wavelet transform based method to this effect was proposed in [5]. Niranjana and Meenakshi proposed a method for ECG signal denoising and Ischemic Event feature extraction using Daubechies wavelets for both healthy signals and abnormal signals [6]. A method to remove baseline wander noise from the ECG signals using independent component analysis is detailed in [7].

Although the DWT is a powerful tool for denoising, it suffers from disadvantages like shift- sensitivity, poor directionality and lack of phase information. These disadvantages restrict its scope for certain signal processing applications. Recent research suggests that frames have very attractive features. Better time frequency localization can be achieved with frames than is possible with bases. Frames provide added degrees of freedom to carry out design, and also numerous methods are available to generate frames.

A wavelet frame can be constructed by iterating a suitably designed oversampled FIR filter bank. A wavelet frame similar to the Daubechies' orthonormal wavelet transform is the Framelet introduced by Selesnick et al $[8,9,10]$. It is also called as the double density discrete wavelet transform because, at each scale, it has exactly twice the number of wavelet coefficients compared to the DWT, thereby achieving lower shift sensitivity than the DWT [11].The dual-tree complex wavelet transform (DT CWT) with improved orthogonality and symmetry properties preserving the key DT CWT advantages of approximate shift-invariance and good directional selectivity in multiple dimensions was presented [12]. Framelet transform has been applied for various applications recently. It has been used for denoising of gray scale images. Higher PSNR values are obtained for such images in the Framelet domain than in the wavelet domain [13]. Framelet is also applied for the segmentation of tubular structures and has outperformed other methods [14]. Other areas in which Framelet has been applied are image inpainting, video enhancement and content based image retrieval [15][16][17]. 
The main contribution in this paper is the denoising of ECG signals in the Framelet transform domain. The rest of this paper is organised as follows: Section 2 explains the Framelet transform. Section 3 describes the image denoising technique using the Framelet transform, followed by the results in Section 4. Section 5 concludes the paper.

\section{THE FRAMELET TRANSFORM}

Any function $f(t)$ can be expanded using a scaling function $\varphi(\mathrm{t})$, and a wavelet function $\psi(\mathrm{t})$. Although Wavelet and Framelet have similar multi resolution capabilities, the wavelet transform uses one scaling function and a wavelet function, while the Framelet transform uses one scaling function $\varphi(\mathrm{t})$, and two wavelet functions $\psi_{1}(\mathrm{t})$ and $\psi_{2}(\mathrm{t})$. By having an additional wavelet function, better time frequency localisation is achieved in the Framelet transform domain.

The scaling and the wavelet functions are obtained through equations (1)-(3) for the low pass filter and the two high pass filters. The filters $h_{i}(n)$ and $h_{i}(-n)$ should satisfy the perfect reconstruction condition. Let $\mathrm{K}_{\mathrm{i}}$ denote the number of zeros $\mathrm{H}_{\mathrm{i}}$ $\left(\mathrm{e}^{\mathrm{jw}}\right)$ has at $\omega=0$, where $\mathrm{i}=1,2$. The $\mathrm{z}$-transform of each $\mathrm{h}_{\mathrm{i}}(\mathrm{n})$ is given as,

$$
\begin{gathered}
H_{0}(z)=Q_{0}(z)(z+1)^{K_{0}} \\
H_{1}(z)=Q_{1}(z)(z+1)^{K_{1}} \\
H_{2}(z)=Q_{2}(z)(z+1)^{K_{2}}
\end{gathered}
$$

$\mathrm{K}_{0}$ gives the degree of polynomials represented by the integer translations of $\varphi(t)$, and gives an idea about the smoothness of $\varphi(t)$. The number of zero moments of the wavelet filters is given by $K_{1}$ and $K_{2}$. A higher degree of smoothness can be achieved by making $\mathrm{K}_{0}$ greater than the other two values. The values for the shortest analysis filters $h_{0}(n), h_{1}(n)$ and $h_{2}(n)$ are shown in Table 1.

Table 1. Set of analysis filters

\begin{tabular}{|c|c|c|}
\hline $\mathbf{h}_{\mathbf{0}}(\mathbf{n})$ & $\mathbf{h}_{\mathbf{1}}(\mathbf{n})$ & $\mathbf{h}_{\mathbf{2}}(\mathbf{n})$ \\
\hline 0.076223 & -0.020547 & -0.027160 \\
\hline 0.349088 & -0.094105 & -0.124388 \\
\hline 0.602089 & -0.122897 & -0.130165 \\
\hline 0.441941 & 0.061353 & 0.742137 \\
\hline 0.060823 & 0.606332 & -0.460423 \\
\hline-0.083923 & -0.311319 & \\
\hline-0.032029 & -0.118815 & \\
\hline
\end{tabular}

The scaling function and the two wavelet functions are defined by Equations (4) and (5).

$$
\begin{gathered}
\Phi(t)=\sqrt{2} \sum_{n} h_{0}(n) \Phi(3 t-n) \\
\Psi_{i}(t)=\sqrt{2} \sum_{n} h_{i}(n) \Phi(3 t-n), i=1,2
\end{gathered}
$$

The coefficients in different sub bands of the Framelet transform are correlated. Therefore, changes in one coefficient can be compensated by its related coefficient in the other sub band. This property helps to remove the noise more effectively from the corrupted signal. The generators of a Framelet frame with parameters $\mathrm{K}_{0}=5$, and $\mathrm{K}_{1}=\mathrm{K}_{2}=2$ are shown in Fig. 1.
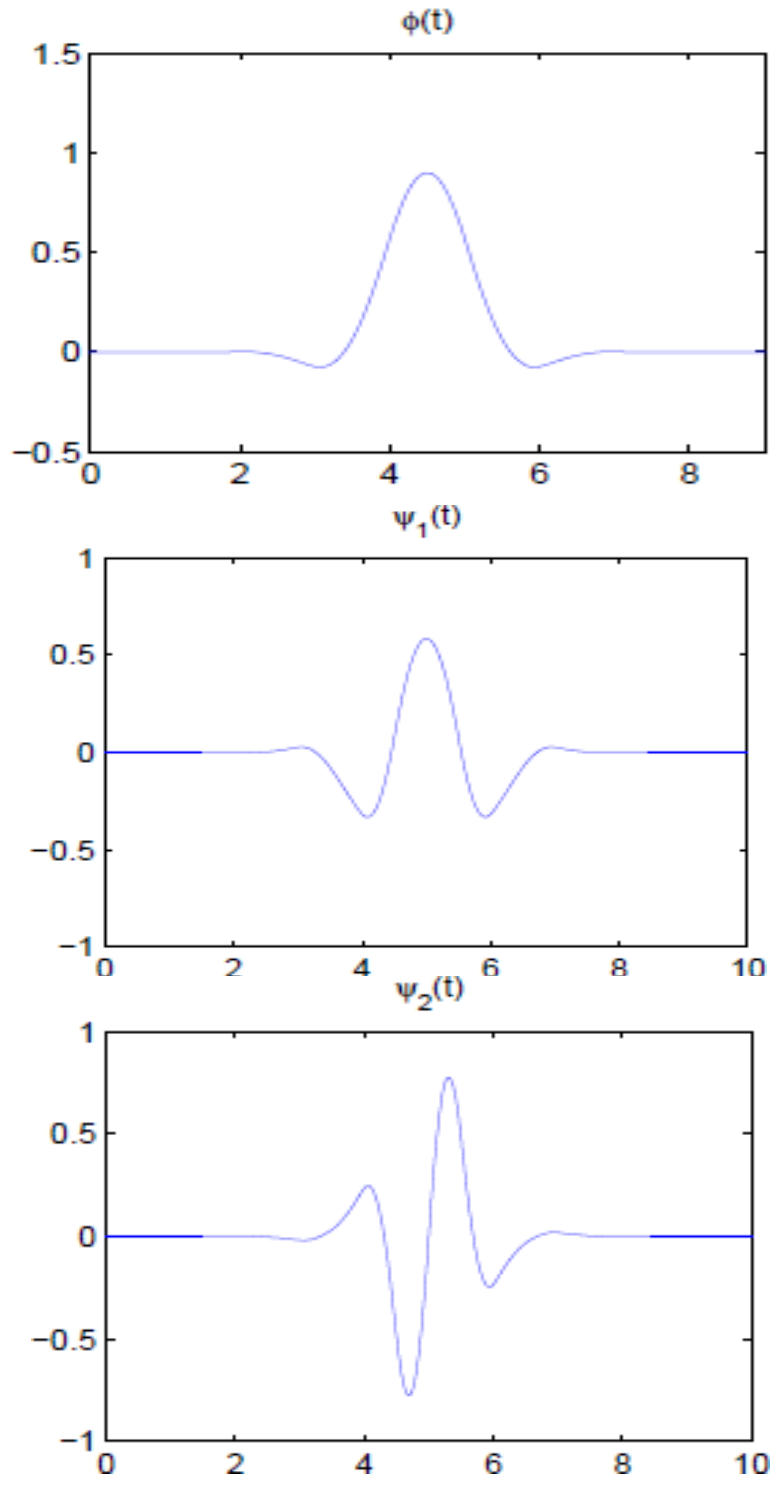

Fig. 1: Generators of a Framelet frame

\section{IMAGE DENOISING USING THE FRAMELET TRANSFORM}

In the proposed denoising system shown in Fig 2, the Framelet transform is used for decomposing the ECG signals. The Framelet transform is implemented using the analysis and synthesis filter banks. The analysis filter bank consists of three analysis filters; one low pass filter denoted by $h_{0}(n)$ and two high pass filters denoted by $h_{1}(n)$ and $h_{2}(n)$. As the input ECG signal travels through the system, the analysis filter bank decomposes it into three sub bands, each of which is then down-sampled by a value of two. The sub bands decomposed from the Framelet transform are then thresholded. These coefficients are then passed on to the inverse Framelet transform which makes use of the synthesis filter bank in order to reconstruct the denoised signal. Fig.3 shows the analysis filter bank used by the Framelet transform. 


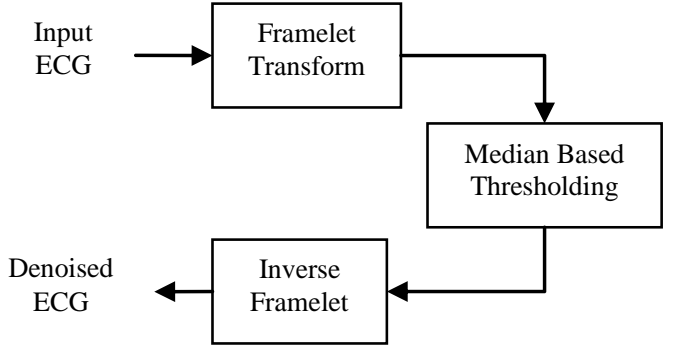

Fig 2: Framelet based denoising system

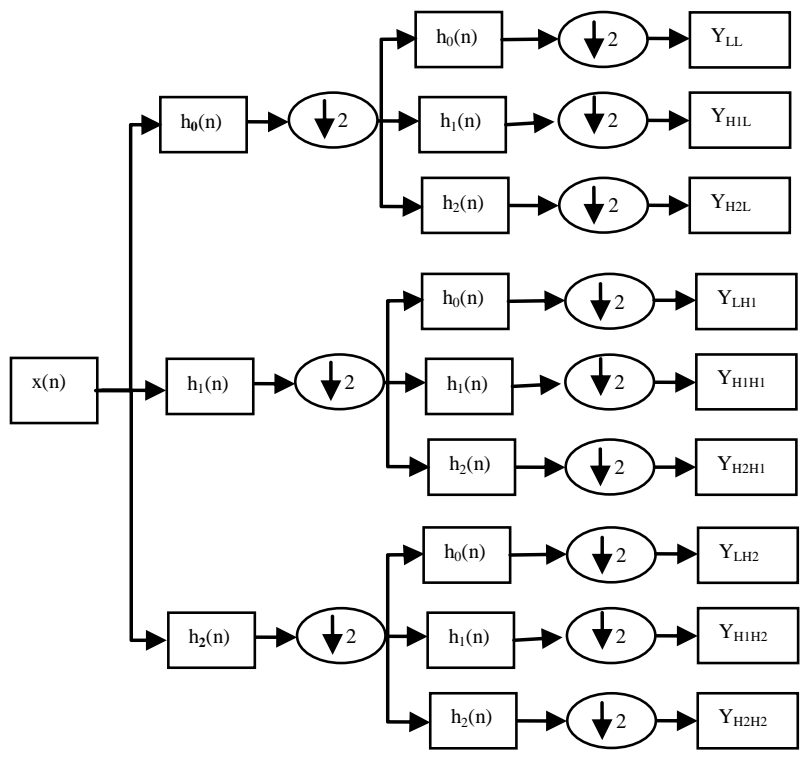

Fig 3: Analysis filter banks

\subsection{Noisy Signal Generation}

In order to generate a noisy signal, several healthy ECG signals are obtained from the Physiobank database first [18]. In general, ECG signals are affected by various kinds of noises like the Gaussian noise, power line interference, baseline drift, and muscle contraction. Here, the focus is on the removal of the Gaussian noise. Therefore, a noisy signal is generated by adding the Gaussian noise to the healthy signal. The probability density function of a Gaussian random variable $\mathrm{x}$ is given by Equation (6)

$$
p(x)=\frac{1}{\sigma \sqrt{2 \pi}} e^{\frac{1}{2}\left(\frac{x-\mu}{\sigma}\right)^{2}}
$$

where, $\mu$ is the mean and $\sigma$ is the standard deviation.

\subsection{Denoising}

The denoising operation is performed in the Framelet domain. The noisy ECG signal decomposed using the Framelet transform get distributed into three different sub bands in the Framelet domain. For further processing, the decomposed sub band signals are stored in the cell array and then passed on to the thresholding function which involves two processes: first, calculation of the threshold value $(\mathrm{T})$ and then comparing the value of $\mathrm{T}$ with the Framelet coefficients. Typically used methods for denoising the ECG signal are briefly explained as follows:

\subsubsection{SureShrink}

SureShrink is an adaptive thresholding method to compute a separate threshold for each sub band, based on Stein's unbiased estimator for risk (Sure). The Sure threshold is defined as,

$$
T=\sqrt{2} \log \frac{N \log N}{\log 2}
$$

where $\mathrm{N}$ is number of Framelet coefficients in the particular sub band.

\subsubsection{VisuShrink}

The VisuShrink thresholding uses the Universal threshold proposed by Donoho and Johnston [3]. VisuShrink does not deal with minimizing the mean squared error. It is efficient in the removal of the additive noise but cannot remove the speckle noise. A smooth denoised signal can be obtained by using this method. The VisuShrink threshold is given by,

$$
T=\sigma \sqrt{2} \log N
$$

where $\mathrm{N}$ is the number of samples in the signal and $\sigma$ is the noise variance.

\subsubsection{Median based Threshold}

The median based threshold is adaptive to the sub band noise signals and obtains the denoised signal very close to the noisefree signal and takes less risk than the universal threshold. The threshold for median based method is given by,

$$
T=\sigma \sqrt{2} \log N
$$

where $\sigma$ is the median of the Framelet coefficients within each sub band.

Thresholding can be applied as a hard threshold or soft threshold. In a hard threshold, all Framelet coefficients greater than a given threshold value are retained, and the remaining coefficients are made zero. In soft thresholding, if a Framelet coefficient is greater than the given threshold, then the coefficient is subtracted from threshold.

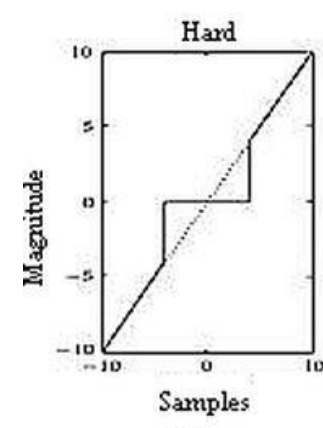

(a)

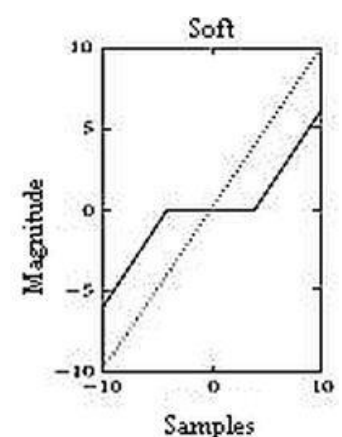

(b)
Fig 4: (a) Hard Threshold (b) Soft Threshold

This operation shrinks coefficients above the threshold in absolute value. If coefficients are less than the threshold, they are added to the threshold. All coefficients equal to the threshold remain unchanged. Fig 4 shows the behavior of the two thresholding methods.

\section{RESULTS}

In order to evaluate the results, the ECG signals are corrupted by adding the Gaussian noise. Each signal is having duration of 1 minute. The percentage of Gaussian noise added to the noise free ECG signal is varied. Experiments were conducted for various noise levels from $0 \%-50 \%$. 


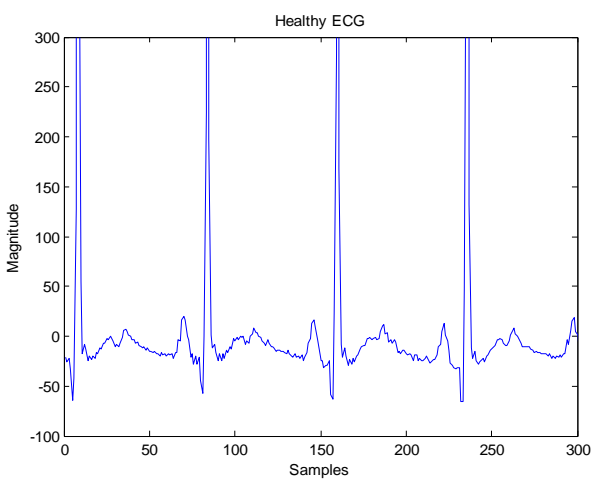

(a)

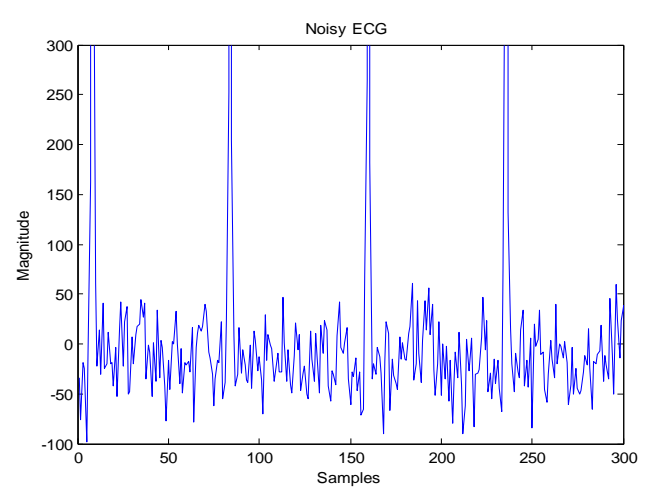

(b)

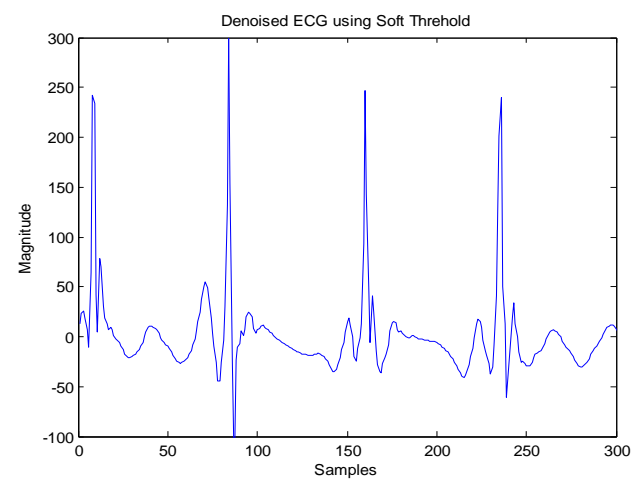

(c)

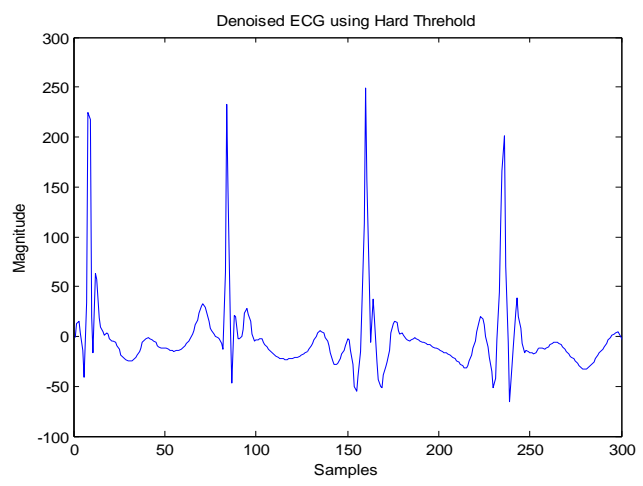

(d)

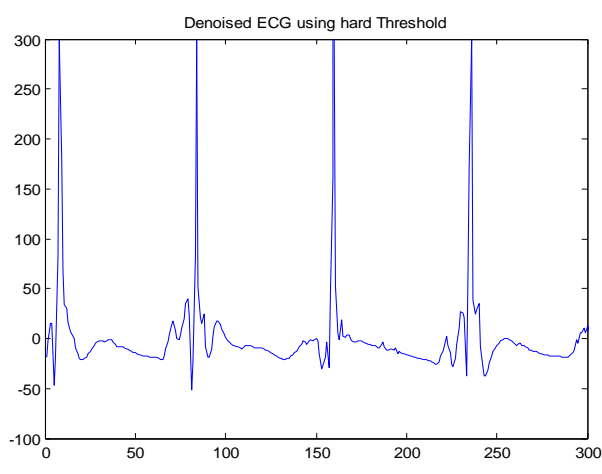

(e)

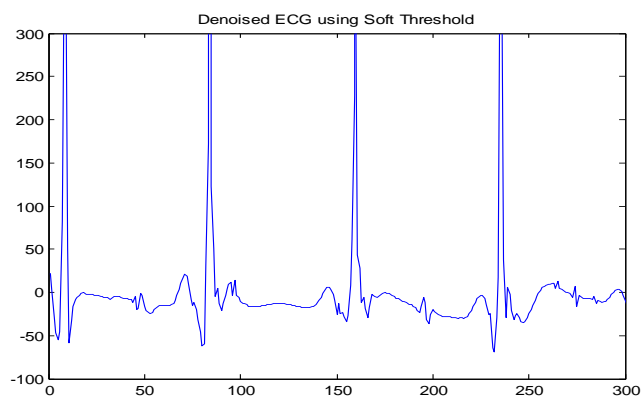

(f)

Fig 5: (a) Healthy ECG Signal (b) ECG Signal with $30 \%$ noise (c)Denoised ECG signal in DWT domain with soft threshold (d) Denoised ECG signal in DWT domain with hard threshold (e) Denoised ECG signal in Framelet domain with hard threshold (f) Denoised ECG signal in Framelet domain with soft threshold

The noisy signals are first decomposed both in the wavelet and the Framelet transform domains using single level decomposition. Then, denoising is conducted using Sureshrink and the proposed median based thresholding method. However, it is observed that the signal denoised after single level decomposition was not denoised properly and had poor visual quality. Therefore, multi level decomposition at levels 2,3 and 4 was conducted and the denoising procedure was repeated in both domains. With a four level decomposition, the median based thresholding method gave a denoised signal of better visual quality in the Framelet domain than in the wavelet domain. A sample ECG signal and its denoising results in wavelet and Framelet domains are shown in Fig 5.

The quality of the denoised signal is measured by calculating the Signal-to-Noise ratio (SNR), which is given by Equation (10).

$$
S N R(d B)=20 \log _{10} \frac{\sigma}{\sigma_{n}}
$$

where, $\sigma$ is the standard deviation of the denoised signal and $\sigma_{\mathrm{n}}$ is the standard deviation of the noise. A comparison of the SNR values obtained in both domains for hard and soft thresholding methods are shown in Table 2. It is inferred that the median based technique using soft thresholding method in the Framelet transform domain yields better SNR than the Sureshrink technique using soft thresholding method in the wavelet transform domain. 
In order to demonstrate the change in the SNR values in the Framelet and the wavelet domains, a large set of noisy signals are generated with the noise variance level at $30 \%$. The SNR values obtained for these signals for the wavelet and the Framelet technique using soft threshold are shown in Fig 6. The superior SNR values in the Framelet domain are due to the usage of two high frequency filters and the resultant improvement in the frequency localization.

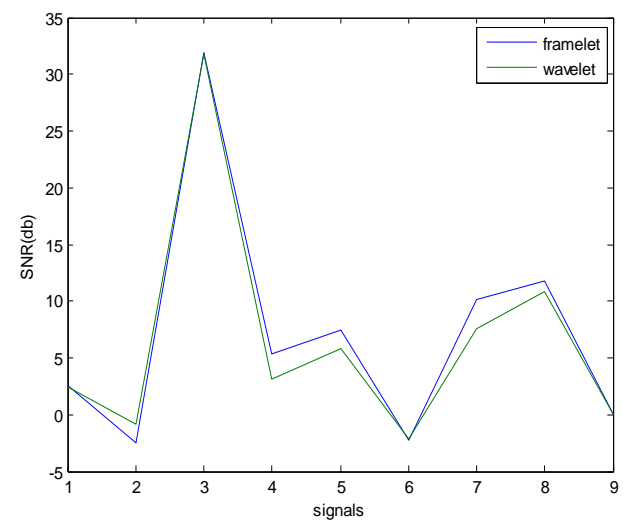

Fig 6: SNR values in Framelet and Wavelet domain with soft thresholding at noise level $=30 \%$

Table 2. Comparison of SNR of various ECG signals fordifferent noise levels

\begin{tabular}{|c|c|c|c|c|c|}
\hline \multirow{3}{*}{ Signal } & \multirow{3}{*}{$\begin{array}{l}\text { Noise } \\
\text { Level } \\
\text { Varian } \\
\text { ce }(\%)\end{array}$} & \multicolumn{2}{|c|}{$\begin{array}{c}\text { Wavelet } \\
\text { (Sureshrink) }\end{array}$} & \multicolumn{2}{|c|}{$\begin{array}{c}\text { Framelet } \\
\text { (Median based) }\end{array}$} \\
\hline & & $\begin{array}{c}\text { Hard } \\
\text { Thresh } \\
\text { old }\end{array}$ & $\begin{array}{c}\text { Soft } \\
\text { Thresh } \\
\text { old }\end{array}$ & $\begin{array}{l}\text { Hard } \\
\text { Thres } \\
\text { hold }\end{array}$ & $\begin{array}{c}\text { Soft } \\
\text { Thresh } \\
\text { old }\end{array}$ \\
\hline & & $\begin{array}{l}\text { SNR } \\
\text { (dB) }\end{array}$ & $\begin{array}{l}\text { SNR } \\
\text { (dB) }\end{array}$ & $\begin{array}{l}\text { SNR } \\
(\mathrm{dB})\end{array}$ & $\begin{array}{l}\text { SNR } \\
(\mathrm{dB})\end{array}$ \\
\hline \multirow{3}{*}{$\mathrm{a} 04 \mathrm{~m}$} & 20 & 8.070 & 8.041 & 6.778 & 9.689 \\
\hline & 30 & 3.871 & 3.121 & 3.486 & 5.837 \\
\hline & 40 & -0.439 & 0.053 & 1.187 & 2.910 \\
\hline \multirow{3}{*}{$\mathrm{a} 05 \mathrm{~m}$} & 20 & 9.956 & 10.450 & 7.763 & 11.681 \\
\hline & 30 & 5,618 & 5.870 & 4.748 & 7.928 \\
\hline & 40 & 2.572 & 2.315 & 2.030 & 5.245 \\
\hline \multirow{3}{*}{$\mathrm{a} 07 \mathrm{~m}$} & 20 & 12.38 & 12.337 & 10.490 & 13.628 \\
\hline & 30 & 8.103 & 7.537 & 6.590 & 9.760 \\
\hline & 40 & 4.492 & 4.754 & 4.374 & 6.735 \\
\hline
\end{tabular}

\section{CONCLUSION}

An approach for denoising the ECG signal in the Framelet domain is implemented in this paper. The noisy ECG signal is decomposed using the Framelet transform. The decomposed signal is then denoised in the Framelet domain using a new median based thresholding technique. This technique also shows that multilevel Framelet decomposition with soft thresholding gives better results than the hard thresholding. Performance evaluation shows that the SNR values obtained using the median based thresholding are better than that of the SureShrink algorithm. Experimental results further show that the Framelet based denoising technique is able to achieve SNR value superior than the wavelet based denoising techniques. As a Future work this can be further be applied to analyze various types of images and videos. Other denoising techniques also can be applied further to see the comparative effects of these data types.

\section{REFERENCES}

[1] Nagendra, H, Mukherjee, S. and Vinod kumar. 2011 Application of Wavelet Techniques in ECG Signal Processing: An Overview, International Journal of Engineering Science and Technology, 3(10): 7432-7443.

[2] Poornachandra, S.and Kumaravel, N. 2006. SubbandAdaptive Shrinkage for Denoising of ECG Signals, EURASIP Journal on Applied Signal Processing, doi:10.1155/ASP/2006/81236: 1-9.

[3] Donoho, D.L. 1995. De-noising by soft-thresholding. IEEE Transactions on Information Theory, 41(3): 613627.

[4] Mikhled Alfaouri and Khaled Daqrouq 2008. ECG Signal Denoising By Wavelet Transform Thresholding, American Journal of Applied Sciences, 5 (3): 276-281.

[5] Karthikeyan, P. and Yaacob, S. 2012. ECG Signal denoising using Wavelet Thresholding Techniques in Human Stress Assessment, International Journal On Electrical Engineering and Informatics, 4(2): 306-319.

[6] Niranjana H S Murthy and M Meenakshi. 2013. ECG Signal Denoising and Ischemic Event Feature Extraction using Daubechies Wavelets. International Journal of Computer Applications, 67(2): 29-33.

[7] Nevi Jain and Devendra Kumar Shakya. 2014. Denoising Baseline Wander Noise from Electrocardiogram Signal using Fast ICA with Multiple Adjustments. International Journal of Computer Applications 99(2): 34-39.

[8] Selesnick, I.W. and L. Sendur. 2000. Iterated oversampled filter banks and wavelet frames. In: Wavelet Applications in Signal and Image Processing VIII, San Diego. Proceedings of SPIE, Vol. 4119.

[9] Selesnick, I.W., 2001. Smooth wavelet tight frames with zero moments. Applied and Computational Harmonic Analysis (ACHA), 10 (2): 163-181.

[10] Selesnick, I.W., 2004. The double-density dual tree DWT. IEEE Transactions on Signal Processing, 52 (5): 1304-1314

[11] Daubechies, B. Han, A. Ron, and Z. Shen, 2003. Framelets: MRA-based constructions of wavelet frames, Applied and Computational Harmonic Analysis, 14: 146.

[12] Kingsbury, N.G., 1998. The Dual-Tree Complex Wavelet Transform: A New Technique for Shift Invariance and Directional Filters. Proceedings of the Eighth IEEE DSP Workshop, Utah, August 9-12.

[13] Sulochana, S. and Vidhya, R. 2012. Image denoising using adaptive thresholding in the Framelet transform 
domain, International Journal of Advanced Computer Science and Applications, 3(9)

[14] Xiaohao Cai, Raymond H.Chan. Serena Morigi and Fiorella Sgallari. 2012. Framelet-Based Algorithm for Segmentation of Tubular Structures, Scale Space and Variational Methods in Computer Vision Lecture Notes in Computer Science Vol.6667: 411-422.

[15] Jian-Feng Cai , Raymond H. Chan, Zuowei Shen. 2008. A framelet-based image inpainting algorithm, Applied and Computational Harmonic Analysis, 24(2): 131-149
[16] R. Chan, L. Shen, and T. Xia. 2007. A Framelet algorithm for enhancing video stills, Appl. Comput. Harmon. Analysis, 23(2): 153-170.

[17] Sulochana,S and Vidhya, R. 2013. Texture Based Image Retrieval Using Framelet Transform-Gray Level Cooccurrence Matrix(GLCM), International Journal of Advanced Research in Artificial Intelligence(IJARAI), 2(2): DOI: 10.14569/IJARAI.2013.020211

[18] Http://www.physionet.org/cgi-bin/atm/ATM 